\title{
Representaciones y existencia matemática
}

\author{
JOSÉ FERREIRÓS \\ Universidad de Sevilla
}

UNO DE LOS PROBLEMAS MÁs DEBATIDOS en Filosofía de las Matemáticas es la espinosa cuestión del significado y alcance de las afirmaciones de existencia que se realizan en el contexto de la matemática moderna. El debate sobre las nociones de 'existencia' y 'verdad' fue central en la famosa crisis de fundamentos que se desarrolló en los años 1920 y 1930, y el desacuerdo respecto a dichas nociones -asociado a fuertes diferencias metodológicas- es quizá la clave para entender el cisma entre los matemáticos 'modernos' y los constructivistas. Aunque no ignoro en absoluto que dicho cisma sigue abierto, en la medida en que algunos grandes matemáticos siguen siendo proclives al constructivismo, ${ }^{1}$ centraré mi atención en la noción de existencia propia de la matemática moderna, hilbertiana, para presentar algunas ideas sobre la conexión que parece darse, en la práctica matemática, entre afirmaciones de existencia consideradas legítimas y representaciones cognitivas adecuadas.

Comenzaremos con algunas aclaraciones relativas a la noción de existencia hilbertiana, seguidas de consideraciones sobre la fuente de las estimaciones de consistencia que se hacen respecto a las teorías de la matemática moderna. Después discutiremos el papel de la comprensión conceptual como base para tales estimaciones, y el lugar que les corresponde a las representaciones en este contexto. El caso principal en el que centraré mi atención es la representación del universo conjuntista que se toma como base en la llamada concepción iterativa de los conjuntos. La importancia de este caso es que a menudo la concepción iterativa se presenta como el trasfondo intuitivo de los axiomas habituales para la teoría de conjuntos.

1 Algunos ejemplos importantes del último medio siglo son Errett Bishop (Foundations of constructive analysis, 1967), Per Martin-Löf (Intuitionistic type theory, 1984), S. Feferman (In the light of logic, 1998). Veáse por ejemplo D. Bridges (2008). 
El viejo y, al decir de Leibniz, laberíntico problema de concebir adecuadamente el continuo, junto con la solución que al mismo dio la teoría de conjuntos, tuvieron un importante papel histórico en replantear y agudizar la cuestión del alcance y significado de las afirmaciones de existencia. Para Dedekind, Cantor y Hilbert, el continuo no es otra cosa que el conjunto $\mathbf{R}$ de los números reales, lo que equivale a analizar el continuo como un conjunto de puntos (o sea, identificar la extensión continua unidimensional con un agregado actualmente infinito de elementos inextensos, contraviniendo la explícita opinión en contra de Aristóteles). Al desarrollar de manera rigurosa esta idea, en sus artículos de 1872, Cantor y Dedekind introdujeron el infinito actual a dos niveles: al nivel de los conjuntos infinitos más simples, totalidades infinitas enumerables como el conjunto $\mathbf{N}$ de los números naturales y el $\mathbf{Q}$ de los racionales; y también al nivel de lo que se llama el conjunto potencia de $\mathbf{N}$ (esto es, el conjunto de todos los subconjuntos de $\mathbf{N}$ ), o el conjunto potencia de $\mathbf{Q}$, que está implícitamente involucrado en los procedimientos empleados por Cantor y Dedekind para definir $\mathbf{R}$ sobre la base de $\mathbf{Q} .^{2}$

Es bien conocido que, con esos avances hacia la matemática moderna, se introdujeron también problemas de muy difícil (si no imposible) solución: el célebre problema del continuo de Cantor no hace más que preguntar algo aparentemente muy simple acerca de los conjuntos de números reales. ¿Qué propiedades de cardinalidad tienen los subconjuntos de $\mathbf{R}$ ? Cantor pensaba que, si un conjunto $\mathrm{C}$ de números reales es infinito, entonces se dará uno de los dos casos siguientes: o bien $\mathrm{C}$ es enumerable, se puede poner en correspondencia uno-a-uno con $\mathbf{N}$; o bien $\mathbf{C}$ tendrá la cardinalidad del continuo, se podrá poner en correspondencia uno-a-uno con $\mathbf{R}$ mismo. Esta es la famosa hipótesis cantoriana del continuo, que ha resultado ser compatible con los axiomas de la teoría de conjuntos (Gödel 1939) pero independiente de ellos (Cohen 1963). Con estos resultados no queda solventado plenamente el problema del continuo, pero una mayoría de expertos desespera de poder encontrar la solución completa. ${ }^{3}$

Cuando Hilbert, en la célebre conferencia 'Sobre los problemas futuros de las matemáticas' (París, 1900), propuso el de Cantor como primer problema de su lista, llamó la atención también sobre una cuestión previa. Era necesario

2 "Sucesiones fundamentales" en el caso de Cantor (2005), "cortaduras" en el de Dedekind (1998). En teoría de conjuntos, es muy importante un tercer nivel del infinito: los grandes cardinales, pero aquí no entraremos en esta cuestión.

3 También hay una minoría de expertos que esperan encontrar una solución unívoca; en su momento, Gödel (1947/64) fue el principal exponente, y en tiempos recientes destaca la figura de Woodin (2001). 
demostrar que todo conjunto admite un buen orden, ya que esto implicaría que la cardinalidad del conjunto $\mathbf{R}$ se corresponde con uno de los "alefs" introducidos por Cantor. ${ }^{4}$ Hilbert, concretando más, decía que debería especificarse "efectivamente" un buen ordenamiento de los números reales. [Se dice que un conjunto $\mathrm{C}$ está bien ordenado cuando sobre él está definida una relación de orden linear o total, de manera que todo subconjunto de $\mathrm{C}$ tiene un primer elemento según ese orden. Esto es lo que sucede con el conjunto $\mathbf{N}$ de los naturales en su orden estándar, pero el orden estándar de $\mathbf{R}$, pese a ser un orden total, no es un buen orden.] Lo que pedía Hilbert, pues, era definir una relación de orden no-estándar sobre $\mathbf{R}$ que le dé a este conjunto una estructura de buen ordenamiento.

En 1904, Zermelo resolvió el problema demostrando el teorema del buen orden, pero su solución no era del tipo que había sugerido Hilbert. El teorema de Zermelo se quedaba muy lejos de indicar "efectivamente" un buen ordenamiento de R: se limitaba a mostrar que la existencia de un buen orden sobre $\mathbf{R}$ es consecuencia lógica del Axioma de Elección y el Axioma del Conjunto Potencia. Enseguida surgieron críticos de este teorema, del axioma de Elección, y de los resultados de existencia similares al de Zermelo (véase Moore 1982, o Ferreirós 1993). Ya años antes, a la vista de un teorema de Hilbert que demostraba la "existencia" de cierto objeto matemático sin dar ninguna indicación de cómo construirlo efectivamente, su colega Paul Gordan escribía con un humor certero: "esto es teología, no matemáticas". Lo mismo pensaron grandes matemáticos, como los analistas franceses, del teorema de Zermelo; y con mayor razón, ya que en este caso (a diferencia del teorema de la base de Hilbert) la distancia entre existencia abstracta y construcción explícita resulta insalvable.

"Existe" quiere decir aquí que, en el universo abstracto que describen (parcialmente) los axiomas de la teoría de conjuntos, hay un objeto dotado de tales y cuales características. El problema de las afirmaciones de existencia se agudiza debido al "platonismo" metodológico de la matemática moderna: la estructura lógica de estas teorías es tal que los objetos estudiados en ellas se tratan como si fueran independientes del sujeto pensante y sus capacidades de facto de construir o definir (Bernays 1935). En particular, algunos de esos objetos no pueden ser especificados explícitamente (por un matemático o por una máquina) con los medios que suministra la teoría.

Aunque no conviene entrar en tecnicismos, indicar que el empleo esencial del Axioma de Elección en el teorema del buen orden proviene precisamente

4 En particular, la hipótesis de Cantor indicada arriba equivale a decir que el cardinal de $\mathbf{R}$ es $\aleph_{1}$ (el primer alef transfinito mayor que el cardinal $\aleph_{0}$ de los conjuntos enumerables). Pero a priori es incluso posible que el cardinal de $\mathbf{R}$ no fuera ninguno de los alefs; el teorema del buen orden excluye esta posibilidad. 
de la imposibilidad de especificar efectivamente (o construir) el objeto buscado en el caso general. Más concretamente, no es posible definir efectivamente un buen orden sobre $\mathbf{R}$ empleando los medios del sistema axiomático ZFC (axiomas de Zermelo-Fraenkel con el axioma de Elección), aún si se añade la hipótesis del continuo generalizada. Este resultado metamatemático fue obtenido por Feferman (1965) empleando una extensión de los métodos de forcing que introdujera Cohen en 1963.

Los matemáticos de tendencia constructivista se niegan a aceptar una noción tan especulativa o "teológica" de existencia, e imponen el requisito de que las afirmaciones de existencia deben ir respaldadas por procedimientos efectivos de construcción o definición del objeto buscado. Sin advertirlo, en 1900 Hilbert había formulado el problema del buen orden de una manera que ponía de relieve las tensiones entre metodologías matemáticas incompatibles. En breve dichas tensiones se hicieron notar y generaron la célebre crisis de fundamentos. Y, como vemos, asuntos relativos al análisis matemático de la idea del continuo, y a las bases axiomáticas y metodológicas de la teoría de conjuntos, están profundamente implicados con el problema de las afirmaciones de existencia en matemáticas.

¿Cabe justificar de alguna manera las afirmaciones de existencia de las teorías matemáticas contemporáneas? Como ya he apuntado, es posible relacionar esta cuestión con la elaboración de representaciones asociadas a dichas teorías y sus objetos. A fin de acercarnos a este punto de vista, empezaremos con algunas consideraciones sobre la visión que propuso Hilbert.

Es famosa la idea hilbertiana de que la consistencia lógica es suficiente para afirmar la existencia matemática: los objetos de que habla una teoría pueden considerarse existentes si y sólo si la teoría es consistente, o sea, está exenta de contradicciones. Como le dijo a Frege: "tan pronto como he establecido un axioma, está dado [vorhanden] y es "verdadero"; o mejor dicho:

Si los axiomas establecidos arbitrariamente no se contradicen entre sí con todas sus consecuencias, entonces son verdaderos, entonces las cosas definidas mediante los axiomas existen. Ese es para mí el criterio de la verdad y la existencia. (carta de 29.12.1899; Frege 1976, 66).

Unos años mas tarde, en carta a Frege del 7.11.1903, Hilbert se expresaba así: 
La laguna esencial en la elaboración tradicional de la lógica, la veo en asumir que un concepto está ya disponible [ein Begriff bereits da sei] si uno puede decir, para cualquier objeto, si cae bajo el concepto o no -cosa que todos los lógicos y matemáticos han asumido hasta hoy-. Esto me parece insuficiente. En su lugar, la cuestión decisiva es el conocimiento de la no contradictoriedad de los axiomas que definen el concepto. (Frege 1976, 80)

Como he tratado de mostrar en otro lugar (Ferreirós 2008), dicha propuesta fue elaborada por Hilbert en respuesta a las antinomias de la teoría de conjuntos, y muestra el influjo residual de ideas logicistas. Pero lo que nos importa aquí es clarificar su concepción, que históricamente está ligada a las ideas de Dedekind y (salvando algunas distancias filosóficas) Cantor, pero alejada de la visión de Frege.

Combinando dos ideas de Hilbert, puede decirse que su planteamiento de la existencia matemática apunta a la existencia ideal de los objetos matemáticos. Pretende un alejamiento de cualquier preocupación ontológica o metafísica, para enfatizar algo así como el carácter de meros entes de razón, "entes del pensamiento" [Gedankendinge] o meras posibilidades lógicas, que tienen los objetos matemáticos. He aquí un tema con cierto aire logicista, que recuerda inmediatamente a Dedekind.

No se le escapaba a Hilbert que la consistencia lógica no implica la verdad tout court; su intención era más bien sugerir una visión minimalista o en cierto sentido "deflacionista" de la verdad matemática.

Ciertamente, la consistencia lógica de un marco teórico entraña su admisibilidad en el dominio del pensamiento. Lo que es lógicamente consistente, puede ser pensado coherentemente. La "existencia" de los objetos matemáticos en tanto "entes del pensamiento" no sería, pues, otra cosa que su existencia relativa a un marco teórico consistente. (El matemático no pretende que el buen orden de $\mathbf{R}$ "exista" en un sentido metafísico o estrictamente realista; el buen orden de $\mathbf{R}$ existe módulo la teoría $Z F C$, relativamente a esta teoría, cuya consistencia presuponemos.) Este tipo de idea fue propuesto nuevamente por Carnap en su 'Empiricism, semantics, and ontology' (recogido en Benacerraf \& Putnam 1983).

Para desarrollar esta visión filosófico-metodológica de las afirmaciones de existencia, y para terminar con la disputa acerca de la admisibilidad de los métodos matemáticos modernos, Hilbert quiso demostrar rigurosamente la consistencia de las principales teorías matemáticas (la aritmética elemental, la teoría de los números reales, la teoría de conjuntos). En esto consistía su célebre programa, su trabajo metamatemático sobre la teoría de la demostración. Con ello habría logrado, según él mismo dijo, eliminar de la faz de la tierra, de una vez por siempre, las dudas escépticas acerca del conocimiento matemáti- 
co. Pero como todos sabemos, el segundo teorema de incompletud de Gödel (también llamado teorema de indemostrabilidad -se entiende que por medios aritméticos- de la consistencia,) dio al traste con sus esperanzas. ${ }^{5}$

Las dudas escépticas siguen vivas. Y sin embargo, más viva está la confianza de los matemáticos en la consistencia de la teoría de los números reales, la teoría de conjuntos, y por supuesto la aritmética elemental. La cuestión es, ¿cuál es la fuente de esa confianza o creencia?

Una vez descartado que la fuente de nuestras convicciones de consistencia sea la verdad tout court (como propuso Frege) o la demostración rigurosa y puramente formal-sintáctica de la consistencia (como propuso Hilbert), no parece haber otra forma de convencerse de la consistencia de una teoría que no sea la comprensión conceptual de la misma, y el propio desarrollo teórico. Cierto que la consistencia formal es una idea mucho más clara, que admite ser precisada absolutamente por medios sintácticos, tolera un tratamiento puramente matemático, y por ello sería de preferir. La idea de comprensión conceptual o captación por el pensamiento se nos antoja vaga y difusa, demasiado humana para que pueda dar un servicio filosóficamente serio. ¿O puede quizá ser analizada con cierta precisión?

Hemos dicho que la consistencia lógica entraña la admisibilidad de un marco teórico, ya que abre la posibilidad de una comprensión conceptual, o sea, de que dicha teoría sea pensada. Inversamente, cabe argumentar que si un marco teórico puede de facto ser pensado coherentemente, entonces la teoría correspondiente es consistente. Es decir, esquemáticamente:

consistencia $\rightarrow$ comprensión posible;

comprensión actual $\rightarrow$ consistencia.

Pero, si pretendemos que este esquema dé algún rendimiento epistemológico, se deberían especificar criterios precisos que nos permitan afirmar que el marco teórico es pensado de modo coherente.

Cuando hablamos de pensar una teoría, se trata de asociar interpretaciones a los términos y relaciones de la misma; el elemento semántico es clave en este caso. ${ }^{6}$ Si hay pensamientos que nos posibilitan una comprensión conceptual de las fórmulas de una teoría, eso quiere decir que hay significados asociados a sus términos y relaciones. Por desgracia, todo esto no es suficiente para decir que

5 No es lugar éste para entrar nuevamente en tema tan conocido. Véanse por ejemplo Sieg (1988), Jané (2006).

6 No se trata aquí principalmente de semántica formal, ya que ésta no es a fin de cuentas sino el recurso a más matemática: la utilización de ciertas teorías matemáticas (presupuestas) como fuente de estructuras que sirven para modelar los enunciados o fórmulas de otra teoría. Así, en el caso de la lógica matemática, la teoría de modelos usual presupone una teoría de conjuntos estándar. 
hemos especificado un criterio medianamente preciso. Pero aquí es, justamente, donde la noción de representación podría venir en nuestro auxilio.

\section{III}

Un caso histórico muy conocido servirá para ejemplificar el papel que llega a desempeñar la representación -y especialmente las representaciones visuales o geométricas- en matemáticas. Como es bien sabido, la fundamentación del concepto de número encontró dificultades serias, hasta tiempos sorprendentemente recientes, con los números complejos. Matemáticos como Gauss y Riemann, ya en pleno siglo xIx, fueron todavía pioneros en defender la plena y total equiparación de los números complejos (antes llamados imaginarios) con los reales. No hubo tanta dificultad con respecto a los números negativos, a pesar de frecuentes discusiones al respecto, porque en este caso era fácil encontrar representaciones como la del debe y haber, o la adición de segmentos dirigidos sobre una recta, que permitían asociar significados y pensamientos al cálculo con dichos números. Pero la introducción de los "imaginarios" fue hija de puras manipulaciones simbólicas que resultaba bastante difícil interpretar. ${ }^{7}$

Entre los años 1799 y 1831, diversos matemáticos desde Argand y Wessel hasta Peacocke y Gauss presentaron en forma rigurosa la forma de representar dichos números en el espacio euclideo bidimensional, o sea, en el plano. Se extendía con esto la representación de los números habituales en la recta real, de tal modo que se asignaba una interpretación geométrica no sólo a cada número (correspondiendo $a+b i$ al punto del plano con coordenadas cartesianas $\langle a, b\rangle$ ), sino también a las operaciones de sumar y multiplicar números complejos. Con ello se lograba un perfecto isomorfismo entre el cálculo con números complejos y una cierta estructura geométrica bidimensional, y en esto consistía la famosa representación geométrica.

En el proceso, el número $i$ dejaba de ser visto como una misteriosa "cantidad' que multiplicada por sí misma arrojaba el resultado -1, para ser identificada con la "unidad lateral" (como dijo Gauss), o sea: la unidad sobre el eje de las y. Tal como enfatiza Miguel de Guzmán:

se puede decir con razón que, en el caso de los números complejos, esta visualización (Argand, Gauss) fue lo que hizo posible vencer la fuerte oposición de la comunidad matemática al dar carta de ciudadanía a los números complejos. ( $E l$ rincón de la pizarra: ensayos de visualización en análisis matemático.)

7 Sobre la historia de los complejos, Flament (2003); sobre el caso particular de Gauss, Ferreirós (2003). 
$\mathrm{Y}$ en efecto, fue imprescindible resolver el problema del significado y la interpretación de los números complejos y sus operaciones, para que finalmente se abriera la vía al enorme desarrollo que supuso el análisis de variable compleja, y también a la teoría de números extendida (enteros de Gauss y otros sistemas), las álgebras correspondientes, etc.

Veremos que, lejos de tratarse de un simple caso particular, achacable quizá al todavía bajo nivel de desarrollo de las ideas abstractas en los inicios del siglo XIX, este tipo de fenómeno es casi omnipresente en matemáticas. El pensamiento visual-diagramático tiene un carácter fundamental en matemáticas, y sus desarrollos (geométricos, topológicos) intervienen a todos los niveles de complejidad o abstracción. Si se quieren algunos ejemplos avanzados, basta pensar en el papel que desempeñan ciertas ramas de la geometría algebraica en el estudio de la teoría de números (ejemplo famoso es la demostración del gran teorema de Fermat por A. Wiles) o la importancia que tiene la introducción de métodos topológicos en todo tipo de cuestiones matemáticas, desde el análisis (por ejemplo, las superficies de Riemann y sus invariantes topológicos) y la teoría de ecuaciones diferenciales, tan cercana a lo físico y lo espacial, hasta la misma lógica matemática, tan abstracta y alejada. $^{8}$

En la parte final de este trabajo, examinaré el papel de las representaciones en conexión con la propia teoría de conjuntos, que tradicionalmente se ha presentado como el fundamento de la matemática moderna. Sin embargo, antes de pasar a ese tema, es imprescindible atacar una objeción que parece plantear el mayor obstáculo ante el proyecto de hacer valer la importancia de las representaciones en el pensamiento matemático y, en último término, en la justificación de las teorías matemáticas. La objeción no es ni más que menos que una cuestión de circularidad: ¿cómo podría ser relevante algún tipo de representación geométrica relacionada con el marco teórico de los conjuntos, si a la inversa la propia geometría debe fundarse en dicho marco?

Nótese que esta objeción proviene de representarse el conocimiento matemático a la manera de los estudios de fundamentos, desde un punto de vista lógico que busca una reconstrucción sistemática de la totalidad de las matemáticas. Es la metáfora arquitectónica del edificio matemático como, en esencia, reducible a un único fundamento teórico. Insisto en este punto, precisamente porque el enfoque que pretendo desarrollar es otro. Cabe analizar el conocimiento matemático desde un enfoque que privilegia la cognición matemática que elaboran efectivamente los individuos, que de esta manera se mantiene cercano a las prácticas matemáticas concretas, y alejado de las grandes reconstrucciones

8 Este importante tema se discute monográficamente en el reciente libro de Giaquinto (2007). 
reduccionistas y sistematizantes. Tal es precisamente el proyecto de análisis del conocimiento matemático que estoy elaborando. ${ }^{9}$

En este enfoque, la clave para un análisis adecuado del conocimiento matemático es reconocer la interacción e interrelación sistemática entre una pluralidad de prácticas, ligadas a su vez a una pluralidad de recursos cognitivos. La coexistencia de diversos estratos del conocimiento matemático, muy lejos de constituir una excepción, es la regla desde un punto de vista cognitivo o incluso histórico. En cada época histórica coexisten prácticas y marcos teóricos diversos, y lo mismo sucede en la expertise del individuo concreto. $\mathrm{Y}$ es a partir de esta diversidad, pero sobre todo a través de las relaciones sistemáticas y perfectamente comunicables que se establecen en la red de prácticas, como se logra una comprensión conceptual de nuevos conceptos matemáticos (o a veces, también, una reconcepción de conceptos ya conocidos).

Un ejemplo muy sencillo puede ilustrar la idea. La comprensión del número natural involucra toda una serie de prácticas, desde las prácticas elementales, pre-matemáticas, de correlacionar y contar, hasta las prácticas simbólicas de cálculo (en las que se hacen prominentes propiedades algebraicas como la asociatividad, conmutatividad, etc.). A su debido tiempo, y en su caso, el concepto de número natural se ve modificado y refinado gracias a la superposición de un nuevo estrato teórico que está ligado al estudio de la estructura del sistema numérico (infinitud, función sucesor, carácter inductivo). Y el proceso podría incluso continuar a través de nuevas inmersiones de esos conocimientos previos en otros estratos teóricos ligados a prácticas más avanzadas: nuevas síntesis.

Similarmente puede analizarse el entramado de prácticas y estratos cognoscitivos ligados a otros conceptos de número -como los racionales, los reales o los complejos-, a conceptos geométricos, y muy particularmente a toda la gama de híbridos o mixtos tan característicos del desarrollo del pensamiento matemático. Por ejemplo, los híbridos de número y geometría tales como el número real (asociado a la representación de la recta real), el número complejo (asociado al plano y los vectores), la geometría analítica (donde ahora se trata, inversamente, de representaciones y métodos algebraicos asociados a las figuras), las superficies de Riemann (constructos geométrico-topológicos $n$-dimensionales ligados a las propiedades de puntos notables de funciones), y un largo etcétera.

En todos estos casos, encontramos representaciones diversas que entrelazan términos, relaciones, fórmulas y conceptos matemáticos, con elementos tomados las más de las veces de la cognición visual, y de prácticas geométricas mas o menos desarrolladas y abstractas. Y al alejarnos del reduccionismo sistemático,

9 En un libro cuyo título provisional es Mathematical knowledge and the interplay of practices, en preparación. Un trabajo de hace años en esta dirección es Ferreirós (2005). 
al separarnos del enfoque que privilegia lo más general y lo más abarcante, surge la tesis de que la comprensión conceptual emerge de la interacción entre prácticas y/o estratos diversos del conocimiento matemático.

Consideremos ahora, desde este punto de vista, las representaciones asociadas con la teoría axiomática de conjuntos: la célebre concepción iterativa del universo conjuntista, que desde nuestro enfoque puede ser vista también como la "representación cónica" de los modelos de la teoría Zermelo-Fraenkel con Elección $(Z F C)$. Sobre esa base, volveremos finalmente a la cuestión de la consistencia de la teoría $Z F C$ y la existencia de las entidades que la misma analiza y postula. ${ }^{10}$

Comencemos recordando en forma simplificada la secuencia histórica de eventos que condujeron a la concepción iterativa. Esta fue sugerida por primera vez por Gödel en una conferencia de 1933 y sobre todo un artículo de 1947, y por tanto salió a la luz 30 o 40 años después de que comenzara el desarrollo de la teoría axiomática de conjuntos. Nació guiada por toda una serie de resultados avanzados que se habían ido obteniendo en esta teoría. Cuando Zermelo en 1908 estableció su sistema axiomático con vistas a consolidar el teorema del buen orden (1904) y los resultados de Cantor y Dedekind (salvando las paradojas y también las críticas al axioma de Elección), le guiaba por cierto un minucioso análisis de la estructura demostrativa de todos esos resultados. Sin embargo, como él mismo confesó, carecía de una concepción nítida y simple de los conjuntos que se pudiera poner en el lugar de la definición ofrecida por Cantor. En nuestro lenguaje, carecía de representaciones asociadas a la caracterización axiomática de la teoría.

En el desarrollo subsiguiente de la teoría hubo una serie de refinamientos lógicos y axiomáticos, pero sobre todo un proceso de estudio de los modelos de la teoría ZFC (debido en los años 1920 a Skolem, von Neumann, Zermelo) que culminó con la adopción del Axioma de Fundación y el trabajo de Zermelo 'Sobre números límite y dominios conjuntistas' (1930). El axioma de Fundación tiene como consecuencia que todo modelo de ZFC admite ser desarrollado en una jerarquía cumulativa de conjuntos, idea que ya sugirió von Neumann. Pues bien, este resultado interno a la teoría axiomática, visto a la luz de elementos notablemente similares que existían en la Teoría de Tipos russelliana, condujo

10 Sobre la concepción iterativa puede verse el libro de Maddy (1998) y los trabajos clásicos recogidos en Benacerraf \& Putnam (1983). Resulta muy interesante la lectura de dos obras pioneras: Gödel (1933) y Zermelo (1930), así como Gödel (1947/64). 
a Gödel a una concepción relativamente simple de la teoría de conjuntos: la concepción iterativa.

En esta concepción, muy diferente de la idea logicista de los conjuntos, ${ }^{11}$ todo consiste en partir de un dominio dado de objetos y en aplicarles la operación "conjunto de" en forma iterada. Si tomamos como nuestro dominio de partida $\mathrm{V}_{0}$ la totalidad de los números naturales, en el primer paso se conciben dados todos los conjuntos de números naturales, y estos nuevos objetos unidos a los de $\mathrm{V}_{0}$ forman el dominio $\mathrm{V}_{1}$. Ahora, en un segundo paso, es posible formar cualquier conjunto cuyos elementos sean números o conjuntos de números; y la totalidad de estos objetos, junto con los de $\mathrm{V}_{1}$, forma el dominio $\mathrm{V}_{2}$. (Como puede verse los dominios son cumulativos en el sentido de que cada uno abarca a los anteriores.) Similarmente, por iteración de "conjunto de", se forman los dominios $\mathrm{V}_{3}, \mathrm{~V}_{4}$, etc. $\mathrm{Y}$ el proceso admite iteraciones transfinitas, puesto que $\mathrm{V}_{\omega}$ puede ser definido como la unión de todos $\operatorname{los} \mathrm{V}_{n}$ para todo número natural $n$, y de ahí se continúa con $\mathrm{V}_{\mathrm{n}+1}$, que es el dominio de todos los conjuntos cuyos elementos pertenecen a $V_{\omega}$, etc. etc.

Así pues, en la concepción iterativa se aplican dos principios de generación de dominios $\mathrm{V}_{\alpha}$ :

1. si $\alpha$ es un ordinal sucesor, $\alpha=\beta+1$, los elementos de $V_{\alpha}$ son todos los conjuntos cuyos elementos pertenecen a $\mathrm{V}_{\beta}$, esto es, $\mathrm{V}_{\alpha}$ es el conjunto potencia de $\mathrm{V}_{\beta}$;

2. si $\alpha$ es un ordinal límite, entonces $\mathrm{V}_{\alpha}$ es la unión de todos los dominios $\mathrm{V}_{\beta} \operatorname{con} \beta<\alpha$.

Precisamente en virtud de esa extensión transfinita del proceso de iteración, es irrelevante que el dominio de partida $\mathrm{V}_{0}$ tenga infinitos elementos o no: podemos incluso empezar con el conjunto vacío, elaborando una teoría de conjuntos pura. Se entiende así que la definición habitual de los dominios sea la siguiente:

(i) $\mathrm{V}_{0}=\varnothing$

(ii) $\mathrm{V}_{\beta+1}=\wp\left(\mathrm{V}_{\beta}\right)$

(iii) $\mathrm{V}_{\lambda}^{\beta+1}=\cup \mathrm{V}_{\alpha}$ para todo $\alpha<\lambda$, siendo $\lambda$ un ordinal límite.

La clase $\mathrm{V}$ formada por todos esos dominios $\mathrm{V}_{\alpha}$ es la jerarquía de von Neumann o jerarquía cumulativa.

11 La concepción dicotómica, en expresión del mismo Gödel, que está asociada al contradictorio principio de comprehensión irrestricta y a la suposición de un conjunto universal. 


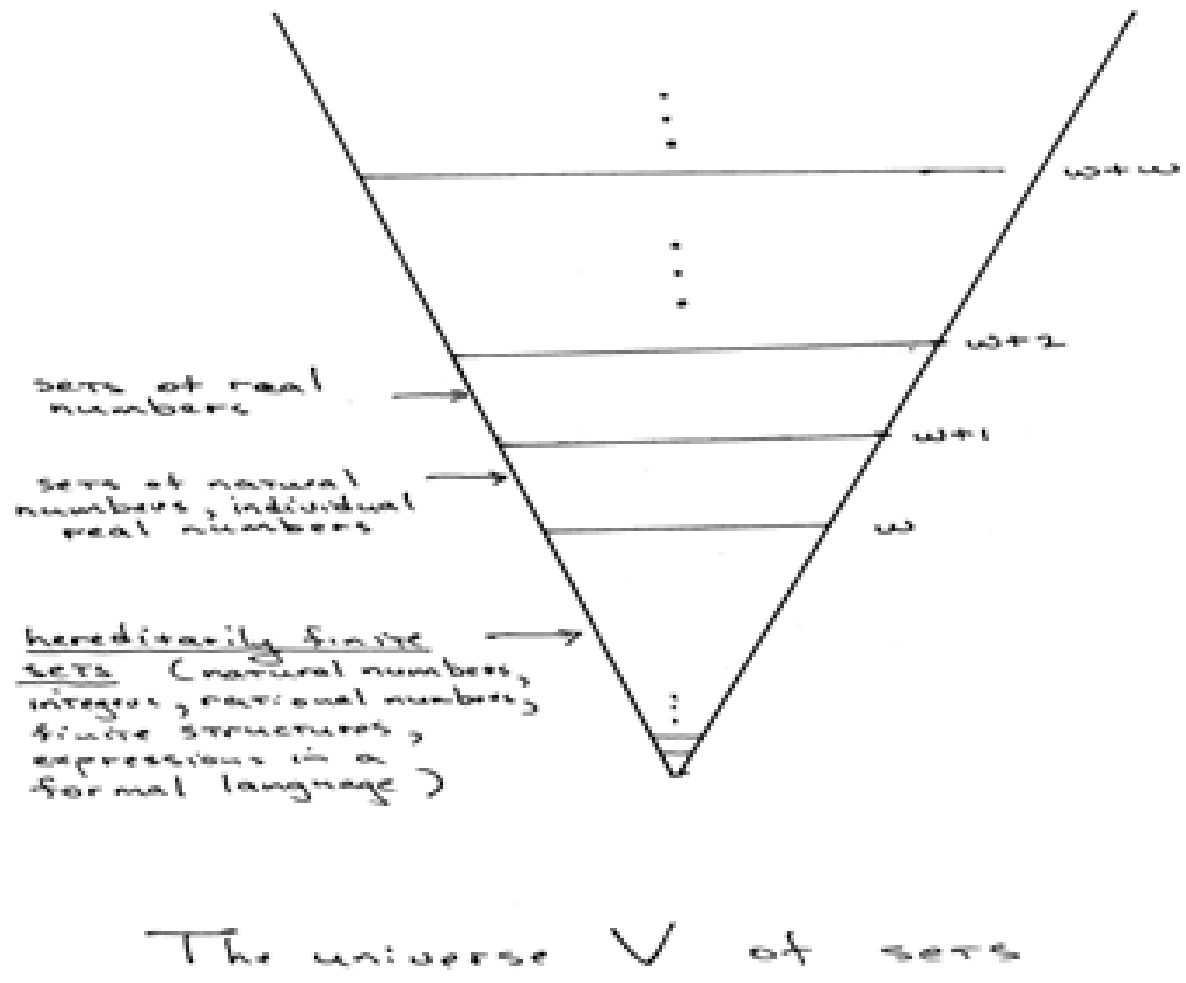

La concepción iterativa tiene muchas ventajas en tanto ofrece una representación del universo conjuntista sobre la base de la cual es posible justificar o al menos motivar buena parte de los axiomas del sistema $Z F C$. No alcanza a justificarlo todo, naturalmente, ya que tiene sus propios presupuestos de partida; pero da una motivación coherente a la teoría axiomática, y supone una representación conceptual unitaria que añadir a la simple enumeración de los axiomas. Se trata además de un enfoque puramente matemático, que conecta con la noción aritmética de iteración y también con representaciones geométricoanalíticas. La imagen geométrica que se emplea con asiduidad es la de un cono que, partiendo del dominio $\varnothing$, asciende hacia nuevos dominios cada vez más grandes (de acuerdo con el célebre teorema de Cantor) en un proceso iterado. El eje del cono lo forman los ordinales transfinitos, de modo que la iteración que se toma por base es transfinita, y la jerarquía admite extensiones sucesivas de acuerdo con hipótesis que entrañan la existencia de grandes cardinales.

Los presupuestos de este enfoque son ante todo dos: A. una concepción decididamente infinitaria que admite sin reservas la existencia de infinitos actuales, y B. el enfoque cuasi-combinatorio que admite subconjuntos ar- 
bitrarios y no meramente los subconjuntos definibles mediante propiedades formalmente especificadas. Dados esos presupuestos, la concepción iterativa motiva muchos axiomas, como sucede con los de Separación, Conjunto Potencia, Unión, Elección (este último, por cierto, resulta totalmente natural a la luz de la concepción que estamos discutiendo). Además, motiva la idea de que el universo de los conjuntos es abierto y, en un sentido bien especificado, esencialmente incompletable y extensible; con lo cual el sistema $Z F C$ no sólo ofrece una solución más o menos provisional al problema de las paradojas, sino toda una explicación del origen de las mismas.

Obviamente, los dos supuestos de base A. y B. no pueden ser justificados a través de la "representación cónica" de los conjuntos, so pena de circularidad. El primero puede restringirse a un axioma del Infinito, asumiendo que el dominio de partida $V_{0}$ es infinito enumerable, tal como hemos hecho en nuestra primera presentación. Pero lo más usual es que se presuponga la escala completa de los ordinales transfinitos, como en las condiciones (i), (ii) y (iii) indicadas arriba, así como la iteración transfinita que subyace a esa definición recursiva. El supuesto B. subyace también a la forma habitual de interpretar el Axioma del Conjunto Potencia, ya que se entiende que $\wp(C)$ tiene por elementos todos los subconjuntos de $C$, definibles o arbitrarios. En suma, estrictamente hablando, ni el axioma del Infinito ni el del Conjunto Potencia, en particular la componente cuasi-combinatoria de éste, admiten ser justificados mediante la concepción iterativa.

Sin embargo, la idea del continuo que es propia del enfoque DedekindCantor motiva el enfoque infinitario. Esa idea consiste en la identificación del continuo con $\mathbf{R}$ y en la concepción de la recta real como agregado infinito actual de puntos. Y tal idea no sólo implica la existencia de conjuntos infinitos, sino que motiva también la admisión de subconjuntos arbitrarios, el cuasi-combinatorismo. El argumento no es difícil de formular, y se basa en considerar las implicaciones del continuo Dedekind-Cantor para la existencia de expansiones decimales arbitrarias (asunto que cabe ligar con la idea de función arbitraria).

En resumidas cuentas, la "representación cónica" o concepción iterativa de los conjuntos supuso una adición significativa a la teoría de conjuntos axiomática, al menos desde el punto de vista cognitivo. Típicamente, al ser expuestos a dicha concepción por vez primera experimentamos la impresión de alcanzar una comprensión conceptual del sistema axiomático; a veces se habla en este sentido de una comprensión "intuitiva". Hay elementos en dicha comprensión que resultan más bien confundentes, y que en un juicio estricto deberían considerarse espurios: sugiere una historia acerca del universo conjuntista entendido como dominio que un sujeto ideal alcanzaría quizá a "construir", siendo así que el punto de vista de la teoría de conjuntos clásica es inevitablemente estático e inabordable desde cualquier punto de vista constructivista. Aquí viene muy a 
cuento la observación de Borges, quien dijo que un hombre inmortal no alcanzaría las dinastías de los transfinitos cantorianos aunque agotara sus eternidades contando; el supuesto "sujeto ideal" que formaría el universo conjuntista tendría obviamente que ser un dios, y no una mera idealización de los sujetos humanos. Pero todo esto no es relevante si adoptamos el enfoque objetivista y estático que es propio de la matemática moderna, al menos en sus versiones clásicas de inicios del siglo Xx.

Si adoptamos un planteamiento no reduccionista, alejado de consideraciones sobre fundamentos últimos, tal como se sugirió en un apartado anterior, es posible ver la concepción iterativa de otra manera. Dicha concepción enlaza con conceptos de iteración aritméticos, extendiéndolos hacia el transfinito en conexión con representaciones geométrico-analíticas tales como la de un cono que se extiende ad infinitum, en una sección finita del cual cabe una infinidad de planos o "dominios", cada uno de ellos actualmente infinito. Y tanto a la luz de las prácticas matemáticas clásicas, anteriores al año 1800, como a la luz de sus revisiones críticas posteriores, tal representación es algo perfectamente coherente, pensable y admisible.

En cuanto a los presupuestos básicos que subyacen a la concepción iterativa, la posición infinitarista y el enfoque cuasi-combinatorio, ya hemos dicho que ambos son naturales una vez admitida la interpretación Dedekind-Cantor del continuo. Sin pretender negar que es cuestión abierta si tenemos una verdadera comprensión conceptual del continuo, lo cierto es que muy pocos pondrían en duda que la interpretación del continuo unidimensional como un agregado actualmente infinito de puntos inextensos es coherente, pensable y admisible. Y esto puede seguir siendo cierto, aún si dicha concepción fuera poco adecuada como interpretación del continuo.

Así las cosas, todo parece apuntar a la conclusión de que la teoría de conjuntos es admisible, en la medida que disponemos de representaciones adecuadas que se asocian con ella y que nos permiten de facto pensar coherentemente dicha teoría. Esta buena comprensión conceptual (más allá del simple hecho empírico de no haber encontrado contradicciones en esta teoría, ni en sus extensiones o derivados, en los 100 años de desarrollo que ya cuenta) es la base conceptual sobre la que parece asentarse, consciente o inconscientemente, la creencia firmemente arraigada en que el sistema $Z F C$ es consistente y nunca dará lugar a nuevas contradicciones.

Y tal como hemos visto al comienzo, siguiendo la línea de pensamiento hilbertiana, las afirmaciones de existencia resultan legítimas en matemáticas en 
la medida en que se derivan del trabajo con teorías consistentes. Así, por fin, sería perfectamente admisible la existencia matemática, ideal, de los objetos conjuntistas (ejemplo notable sería un buen orden de $\mathbf{R}$ ).

Por supuesto, podríamos continuar insistiendo en el entramado de conexiones deductivas entre la teoría de conjuntos y las superestructuras matemáticas "clásicas" que pueden establecerse sobre el fundamento axiomático de ZFC (álgebra, topología, análisis, geometrías). Todo esto forma parte también de la base conceptual sobre la que se asienta la creencia la consistencia de la teoría de conjuntos, mas para abreviar evitaremos entrar en detalles al respecto. ${ }^{12}$

Quizá conviene insistir, para acabar, en dos puntos. Primero, y quizá tan obvio que hace innecesario decirlo, toda la argumentación anterior tiene el carácter frágil y humano de una reflexión sobre la fábrica y los orígenes de nuestro conocimiento, de nuestras prácticas cognoscitivas. Se queda, así, muy lejos de las garantías estrictas que tienen otros resultados que a una visión ingenua podrían parecer emparentados, como es por ejemplo la demostración por Gödel de la imposibilidad de probar aritméticamente la consistencia del sistema $Z F C$, o su demostración de la consistencia relativa del axioma de Elección y la Hipótesis del Continuo con respecto al sistema $Z F$. Y sin embargo, hay algo de satisfactorio en el entrelazamiento muy sistemático, comprensible y enseñable, de los distintos niveles del conocimiento matemático; se trata de algo mucho más sólido que un mero cuento o una metáfora aducida como justificación.

El segundo punto consiste en decir que la comprensión conceptual del tipo que hemos discutido es compatible con la falta de pleno control matemático. En particular, como analizo en otro lugar, el enfoque cuasi-combinatorio no parece admitir formalización, y de ahí se deriva una limitación quizá inevitable de nuestra capacidad de caracterizar el universo conjuntista al que apunta la teoría de conjuntos (conceptual o "intuitiva"). Cabe entender que esta es la situación a la que remite el caso de la Hipótesis del Continuo de Cantor, independiente de $Z F C$ y de los axiomas de grandes cardinales, hasta el punto de que el problema del continuo es considerado por muchos como irresoluble por motivos más hondos que la mera independencia de $Z F C$.

12 Sobre este asunto ya clásico puede verse la obra de Maddy (1998) o trabajos pioneros como Gödel (1947/64). 


\section{REFERENCIAS}

BENACERRAF, P. \& H. PUTNAM (eds) 1983: Philosophy of Mathematics: Selected readings, Cambridge University Press.

BERNAYS, P. 1935: "Sur le platonisme dans les mathématiques", L'Enseignement Mathématique 34. Versión inglesa en Benacerraf y Putnam, op. cit. (1983).

BRIDGES, D. 2008: "Constructive Mathematics", The Stanford Encyclopedia of Philosophy (Fall 2008 Edition), Edward N. Zalta (ed.), URL $=<$ http://plato. stanford.edu/archives/fall2008/entries/mathematics-constructive/>.

CANTOR, G. 2005: Fundamentos para una teoría general de conjuntos, Barcelona, Crítica.

DEDEKIND, R. 1998: ¿Qué son y para qué sirven los números? y otros escritos, Madrid, Alianza Editorial.

FEFERMAN, S. 1965: "Some applications of the notions of forcing and generic sets". Fundamenta Mathematicae vol. 56, pp. 325-345.

FLAMENT, D. 2003: Histoire des nombres complexes: entre algèbre et géométrie, Paris, CNRS éditions.

FREGE, G. 1976: Briefwechsel mit Hilbert, Husserl, Russell sowie ausgewählte Einzelbriefe, ed. Gottfried Gabriel et al., Felix Meiner Verlag, 1980.

FERREIRÓS, J. 1993: El nacimiento de la teoría de conjuntos, 1854-1908, Publicaciones de la UAM.

- - 2003: “Kant, Gauss y el problema del espacio", en A. Durán y J. Ferreirós (eds), Matemáticas y matemáticos, Publicaciones de la Universidad de Sevilla, pp. 105-133.

- - 2005: "Dogmas and the Changing Images of Foundations", Philosophia Scientiae 5, 27-42.

- - 2008: "Hilbert, Logicism, and Mathematical Existente", Synthese. DOI 10.1007/s11229-008-9347-1.

GIAQUINTO, M. 2007: Visual thinking in mathematics: an epistemological study, Oxford University Press.

GÖDEL, K. 1933: "La situación presente en los fundamentos de las matemáticas". La Gaceta de la RSME 9, no. 3, 761-771.

- - 1947/64: “¿Qué es el problema del continuo de Cantor?”, en Obras completas, Alianza Editorial. Versión inglesa en Benacerraf y Putnam, op. cit. (1983).

DE GUZMÁN, M. 1996: El rincón de la pizarra: ensayos de visualización en análisis matemático, Madrid, Pirámide.

JANÉ, I. 2006: "La obra de Gödel en lógica matemática y teoría de conjuntos", La Gaceta de la RSME 9, no. 3, 772-788.

MADDY, P. 1998: Naturalism in Mathematics, Oxford University Press. 
MOORE, G. H. 1982: Zermelo's Axiom of Choice: Its origins and development, Berlin, Springer.

SIEG, W. 1988: "Hilbert's programs: 1917-1922", The Bulletin of Symbolic Logic 5, 1-44.

WOODIN, H. 2001: "The Continuum Hypothesis". Parts I and II, Notices of the Amer. Math. Soc. 48, no. 6 y 7, 567-576 y 681-690.

ZERMELO, E. 1930: "Über Grenzzahlen und Mengenbereiche". Versión inglesa en W. Ewald (ed), From Kant to Hilbert, vol. 2, Oxford University Press, 1996. 
